\title{
Precision genome editing
}

\author{
A machine learning model predicts the genotype of CRISPR-Cas9 gene editing products, thereby enabling precise, \\ template-free correction of disease-associated mutations.
}

G ene editing tools such as CRISPR-Cas9 have fundamentally changed the ways scientists can modify genetic information. CRISPR-Cas9 gene editing works by targeting the protein Cas 9 to a specific target via a guide RNA (gRNA). Once bound to the target, Cas9 cleaves the DNA, which is subsequently repaired by the cell's DNA-repair machinery. Without a template, this repair process is considered random and heterogeneous, which makes it unsuitable for precision editing. However, precision editing is badly needed, both for studies of basic gene function and for the correction of mutations associated with genetic disorders.

Richard Sherwood, along with $\mathrm{PhD}$ student Max Shen, postdoctoral researcher Mandana Arbab, and professors David Gifford and David Liu at Brigham and Women's Hospital, MIT, and the Broad Institute, sought to address this challenge and make template-free genome editing more precise and predictable. "Many groups, including ours, had noticed that the mutated products of CRISPR-Cas9 editing were not random," Sherwood notes. "Up until now that has mainly been a nuisance, but we thought that if Cas9 outcomes were so nonrandom as to be predictable, it would add a new dimension to the use of CRISPR-Cas9 editing."

The researchers made use of the observation that gene editing products were not random by designing inDelphi, a machine learning algorithm to predict editing and design gRNAs with the desired repair genotype. To make inDelphi work effectively, they needed a large set of training data, which Sherwood says was a major challenge of the work owing to the tedious nature of such screens. "We had the idea of cloning a DNA library that combines a gRNA with its target sequence, thereby allowing us to scale up to look at outcomes at thousands of Cas9 target sites with minimal extra effort," Sherwood recalls. This cell-culture-based assay allowed them to determine gene editing outcomes at over 4,000 target sites associated with the human genome, which is more than an order of magnitude greater than the number of sites associated with conventional screens.

Once the training data were in hand, the researchers still needed to optimize inDelphi. Sherwood notes that "even sophisticated

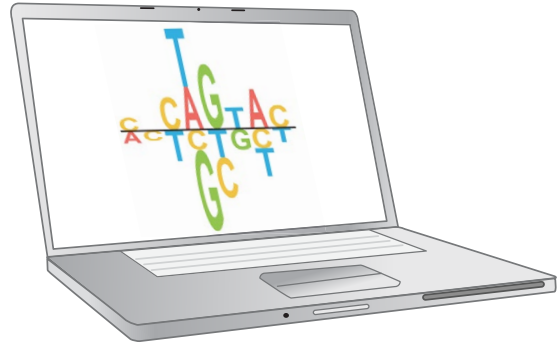

Cartoon depiction of algorithmic prediction of gRNAs. Adapted in part with permission from Shen et al. (2018), Springer Nature. Credit: Marina Corral Spence/Springer Nature

techniques such as deep learning are still quite dependent on the parameters we feed into them." With this in mind, the team developed and tested several versions of the algorithm with various architectures. According to Sherwood, one key to high accuracy was separating the repair outcomes into groups including various deletions and insertions on the basis of their hypothesized repair mechanisms. "Allowing different machine learning sub-algorithms to predict these different repair classes and exchange information with each other in the process helped improve accuracy considerably."

inDelphi identified that $5-11 \%$ of Cas9 gRNAs in the human genome will result in a single, predictable repair product more than half of the time. This means that hundreds of genetic disease alleles can be repaired a majority of the time. To test their trained algorithm, they used inDelphi and their high-throughput library assay to confirm the correction of 183 pathogenic human microduplication alleles in more than half of the edited products. They also designed 14 gRNAs predicted to yield 1-bp insertions more than half of the time in human disease-relevant genes, and confirmed a high success rate. Finally, they showed that they could correct endogenous pathogenic microduplications for Hermansky-Pudlak syndrome and Menkes disease in primary patient-derived fibroblasts. "In essence, people had been using Cas9 as a sledgehammer to break genes indiscriminately, but we show that in certain predictable cases we can use that same hammer to sculpt the genome precisely," Sherwood says.

This work highlights an aspect of gRNA design that has been largely overlooked in favor of other important features such as targeting efficiency and off-target binding. Sherwood calls this area "ripe for optimization" and hopes that repair-outcome precision associated with a given gRNA is kept in mind by researchers designing and testing newly engineered CRISPR-Cas systems-especially in therapeutic settings. "If you could feasibly use two guide RNAs in a patient, and one caused hundreds of different repair outcomes while another predominantly caused a single repair outcome, wouldn't you want to use the guide RNA where there is an element of control over the outcomes?" asks Sherwood.

inDelphi is currently implemented as a user-friendly tool (https://indelphi.giffordlab, mit.edu), and will undoubtedly be a useful tool for guiding genome editing. However, it can still be further improved. "We'd love to make predicting Cas9 outcomes even more precise, allowing us to get a dominant repair product over $90 \%$ of the time," notes Sherwood. The researchers are also interested in studying how inDelphi works in different cell types, such as in neurons and in vivo, where different DNA-repair mechanisms are favored. They would also like to integrate rare large deletions into inDelphi predictions, which Sherwood says would account for all possible editing outcomes. The researchers also believe that the same approach should be extensible to other CRISPR-Cas systems, although Sherwood notes that a new algorithm will probably need to be trained for each nuclease.

This work represents a major advance for CRISPR-Cas9-based genome editing and precision medicine, and could transform the way researchers design these experiments and use these tools.

\section{Rita Strack}

Published online: 20 December 2018 https://doi.org/10.1038/s41592-018-0286-6

Research papers

Shen, M. W. et al. Predictable and precise templatefree CRISPR editing of pathogenic variants. Nature 563, 646-651 (2018). 